\title{
Analysis of Supraglottic Activity During Vocalization in Healthy Singers
}

\author{
Ross M. Mayerhoff, MD; Marco Guzman, SLP; Cristina Jackson-Menaldi, PhD; Daniel Munoz, MD; \\ Jayme Dowdall, MD; Ahmed Maki, DO; Michael M. Johns III, MD; Libby J. Smith, DO; \\ Adam D. Rubin, MD
}

\begin{abstract}
Objectives/Hypothesis: The present study aims to evaluate the degree of anterior-posterior and medial supraglottic laryngeal compression in healthy singers of different voice classifications while singing different pitches, loudness, and phonatory tasks.

Study Design: Prospective study.

Methods: Thirty-six classically trained singers (11 sopranos, 11 mezzo-sopranos, six tenors, and eight baritones) with at least 5 years of voice training and absence of any voice pathology within the past year were included. Flexible endoscopic voice evaluations were recorded and edited to include samples of different pitches, levels of loudness, and phonatory tasks. Sound was removed from the video samples. Two blinded laryngologists were asked to assess medial and anterior-posterior supraglottic compression using a visual analog scale for each sample.

Results: Medial compression was significantly greater in male subjects and specifically tenors during loud phonation, during high pitch, and while phonating the vowel /a/. Anterior-posterior compression was also significantly greater in males and specifically baritones during loud voice production and with phonation of the vowel /a/. No difference was noted in relation to pitch. Correlation between anterior-posterior and medial compression was demonstrated. Medial compression was less severe than anterior-posterior compression.

Conclusions: Supraglottic hyperfunction is present in healthy singers. Recognizing factors that might affect supraglottic hyperfunction in the healthy voice may help us to understand when hyperfunction is truly contributing to a patient's vocal pathology.

Key Words: Laryngeal hyperfunction, supraglottic hyperfunction, laryngoscopy, videostroboscopy, singing voice, classical singers, dysphonia.
\end{abstract}

Level of Evidence: 4.

Laryngoscope, 124:504-509, 2014

From the Department of Otolaryngology-Head and Neck Surgery, Wayne State University, Detroit, Michigan, U.S.A. (R.M.M., C.J.-M.); School of Communication Sciences, University of Chile and Voice Center, Las Condes Clinic, Santiago, Chile (м.G.); Lakeshore Professional Voice Center, Lakeshore Ear, Nose, and Throat Center, St. Clair Shores, Michigan, U.S.A. (C.J.-M., A.D.R.); Department of Network Management, Barros Luco-Trudeau Hospital, Santiago, Chile (D.M.); Department of Otolaryngology, Harvard Medical School, and Department of Otology and Laryngology, Massachusetts Eye and Ear Infirmary, Brigham and Women's Hospital, Boston, Massachusetts, U.S.A. (J.D.); McLaren Oakland, Pontiac, and Michigan State University, East Lansing, Michigan, U.S.A (A.M.); Department of Otolaryngology-Head and Neck Surgery, Emory University School of Medicine, and Emory Voice Center, Atlanta, Georgia, U.S.A. (м.M.J.); Department of Otolaryngology-Head and Neck Surgery, University of Pittsburgh Medical Center, Pittsburgh, Pennsylvania, U.S.A. (L.J.S.); and Department of Otolaryngology-Head and Neck Surgery, University of Michigan Medical Center,, Ann Arbor, Michigan, U.S.A. (A.D.R.)

Editor's Note: This Manuscript was accepted for publication June 28, 2013.

Presented at the Triological Society Combined Sections Meeting, Orlando, Florida, U.S.A., April 10-11, 2013.

The authors have no funding, financial relationships, or conflicts of interest to disclose.

Send correspondence to Adam Rubin, MD, 21000 E. Twelve Mile Road, Suite 111, St. Clair Shores, MI 48081.

E-mail: rubinad1968@gmail.com

DOI: 10.1002/lary.24310

\section{INTRODUCTION}

Several definitions of laryngeal hyperfunction exist, but a recurrent feature in almost all descriptions includes excessive laryngeal musculoskeletal activity, force, or tension. ${ }^{1}$ The basic paradigm for evaluation of laryngeal hyperfunction is to look for compression of the supraglottic structures during phonation. ${ }^{2}$ This supraglottic activity may be divided into two components. Anteriorposterior (A-P) supraglottic activity occurs when the petiole of the epiglottis and the arytenoid cartilages are drawn toward each other. Medial supraglottic activity is characterized by adduction of the false vocal folds. ${ }^{3}$ Hyperfunction is often considered a sign of abuse or misuse of the vocal mechanism. ${ }^{4-8}$ It is commonly reported in patients presenting with voice disorders, particularly nonorganic voice disorders, such as muscle tension dysphonia. ${ }^{9}$ Moreover, the development of many benign lesions on the vocal fold surface has been associated with laryngeal hyperfunction and/or phonotrauma. ${ }^{10}$

Although medial and A-P compression have each been accepted as endoscopic signs of vocal dysfunction, some studies have demonstrated that supraglottic activity may be present in normal speaking ${ }^{3,9,11,12}$ and singing $^{13-15}$ voice production. Lawrence suggests that belting is often typified by a relatively high laryngeal 
position, essentially closed ventricular spaces, constricted pharyngeal diameters, an epiglottis tilted over the larynx, and an elevated tongue base. ${ }^{13}$ Yanagisawa et al. showed that aryepiglottic constriction was present in twang, belting, and operatic voice production. ${ }^{14}$ Pershall and Boone reported that most subjects demonstrated laryngeal supraglottic constriction in different pitches throughout the entire vocal range. ${ }^{15}$

There is also empirical evidence that A-P compression may contribute to an acoustic advantage with regard to voice quality if singers can use this feature with no ill effects. Vocal tract adjustments such as laryngeal rise and aryepiglottic and medial laryngeal constrictions are frequently found during "bright" singing voice quality. ${ }^{16}$ Narrowing of the aryepiglottic orifice results in greater acoustic energy. ${ }^{14}$ Sundberg $^{17}$ proposed that the singer's formant (a prominent spectrum envelope peak near $3 \mathrm{kHz}$ associated with the "ringing" voice quality in classical singers) is produced when the crosssectional area of the larynx is less than one-sixth that of the pharynx. The larynx creates a tube that acts as a separate resonator, making the voice carry over the presence of a loud orchestral accompaniment. ${ }^{17}$

Although previous studies have demonstrated that supraglottic hyperfunction may not be pathologic during singing, they have included small numbers of subjects, and none has evaluated specific variables that may affect the degree of hyperfunction. The present study aims to evaluate the degree of A-P and medial supraglottic laryngeal compression in healthy singers of different voice classifications during different pitches, loudness, and phonatory tasks. Recognizing factors that affect supraglottic hyperfunction in the healthy voice may help us to understand when hyperfunction is truly dysfunctional.

\section{MATERIALS AND METHODS}

\section{Participants}

This study was reviewed and approved by the St. John Hospital Institutional Review Board. Informed consent was obtained from 36 adult classical singers (23 women and 13 men). The average age of this subject set was 39.45 years (35 for females, 43 for males), with a range of 19 to 62 years. Voice types were as follows: 11 sopranos, 11 mezzo-sopranos, 6 tenors, and 8 baritones. We were unable to recruit enough altos and basses to put them in distinct groups for statistical purposes. Therefore, we included altos with mezzo-sopranos and basses with baritones. Inclusion criteria for this study included: 1) no history of voice problems in the past year, 2) no vocal cord pathology, and 3) at least 5 years of classical singing training. The average length of voice training was 9.2 years, with a range of 5 to 20 years. Participants were recruited from three different professional choirs and one department of music at an undergraduate institution. All were asked to undergo flexible laryngoscopy (Pentax VNL-1170K; KayPENTAX, Lincoln Park, NJ) with specific voice tasks (see below). Rigid videostroboscopy (Digital Videostroboscopy System 9295; KayPENTAX) was also performed to confirm the absence of laryngeal pathology. All of the endoscopic laryngeal examinations were performed by the senior author (A.D.R.), who is a fellowship-trained laryngologist. Intranasal topical anesthesia was used (oxymetazoline/tetracaine, 50:50). Although 40 singers were recruited, four singers did not meet the inclusion criteria due to vocal fold pathology found at the time of endoscopy.

\section{Phonatory Tasks}

During the flexible endoscopic examination, each participant was instructed to perform two different singing tasks: 1) to sing sustained vowels $(/ \mathrm{i} /, / \mathrm{a} /, / \mathrm{u} /)$ and 2$)$ to sing the song "Happy Birthday." All of the singers sang in an operatic style. Participants were asked to produce each phonatory task at three loudness levels (pianissimo, mezzo-forte, and fortissimo). Loudness was subjectively controlled by the singers and two of the authors (A.D.R. and C.J.-M.). Subjects were also asked to produce the sustained vowels at three different pitches for each loudness level: A2 $(110 \mathrm{~Hz}), \mathrm{A} 3(220 \mathrm{~Hz})$, and F\#4 $(370 \mathrm{~Hz})$ for baritones; C3 $(131 \mathrm{~Hz}), \mathrm{C} 4(262 \mathrm{~Hz})$, and A4 $(440 \mathrm{~Hz})$ for tenors; A3 $(220 \mathrm{~Hz}), \mathrm{A} 4(440 \mathrm{~Hz})$, and F\#5 $(740 \mathrm{~Hz})$ for mezzo-sopranos; and C4 $(262 \mathrm{~Hz}), \mathrm{C} 5(523 \mathrm{~Hz})$, and A5 $(880 \mathrm{~Hz})$ for sopranos. We chose a low pitch, a middle pitch, and a high pitch for each voice classification. The low and middle pitches were chosen to be below the passaggio, whereas the high pitch has to be above the passaggio. For example, for tenors, C3 is a low pitch, C4 is a middle pitch, and A4 is a high pitch (which is also higher than the passaggio). The musical key of "Happy Birthday" was adapted to each singer's vocal classification: $E$ major for mezzo-sopranos and baritones, and G major for sopranos and tenors. Careful control of pitch throughout the sequence was performed. An electronic keyboard was used to give and control the pitch, which was monitored aurally by one of the experimenters, a PhD-trained voice pathologist and singingvoice specialist (c.J.-M.).

\section{Laryngoscopic Evaluation}

Each recording was edited into 30 short clips separating the vocal tasks (three vowels at three different pitches, at three different loudness levels; and "Happy Birthday" at three different loudness levels). Each clip was assigned a random number. All sound was removed, and the clips were copied onto a DVD. Two fellowship-trained laryngologists (M.M.J. and L.J.s.) blinded to the purpose and nature of the study were asked to review the clips and rate the degree of both medial and A-P laryngeal compression on a $100-\mathrm{mm}$ visual analogue scale (VAS). They were instructed to evaluate each clip as a whole and could review each clip as many times as desired. Once the VAS evaluations were returned, measurements were taken of the distance from the "no compression" side of the VAS to the reviewer's mark (the larger the measurement, the greater the perceived degree of compression).

\section{Statistical Analysis}

Descriptive statistics were calculated for the variables, including mean and standard deviation. A multivariate linear mixed regression model to obtain intraclass correlation coefficient (ICC) to assess inter-rater agreement, controlled by intensity and pitch, was conducted. One-way analysis of variance (ANOVA) or $t$ test for univariate analysis for both medial laryngeal compression and A-P laryngeal compression by loudness level, pitch, gender, voice classification, and phonatory task was used. If there were significant differences in the variables, they were included in a multivariate regression analysis that considers both types of laryngeal compression as dependent variables, and loudness, pitch, gender, voice classification, and phonatory task as predictors. A $t$ test was used to compare overall medial and A-P compression VAS scores. Linear correlation analysis using Pearson linear correlation coefficient $(r)$ between medial 


\begin{tabular}{lccr}
\hline \multicolumn{4}{c}{ TABLE I. } \\
Visual Analogue Scale Scores in Relation to Gender. \\
\hline Score & Male & Female & $P$ \\
\hline Medial & $19.39 \pm 10.39$ & $12.32 \pm 7.22$ & $<.0001$ \\
Anterior-posterior & $30.35 \pm 24.15$ & $24.51 \pm 19.73$ & .0001 \\
\hline \hline
\end{tabular}

TABLE II.

Visual Analogue Scale Scores in Relation to Loudness Level.

\begin{tabular}{lcccc}
\hline Score & $\mathrm{pp}$ & $\mathrm{mf}$ & $\mathrm{ff}$ & $\mathrm{P}$ \\
\hline Medial & $13.64 \pm 8.16$ & $14.45 \pm 8.08$ & $17.32 \pm 10.99$ & $<.0001$ \\
$\begin{array}{l}\text { Anterior- } \\
\text { posterior }\end{array}$ & $23.30 \pm 20.59$ & $25.85 \pm 21.05$ & $31.44 \pm 22.88$ & $<.0001$ \\
\hline
\end{tabular}

$\mathrm{ff}=$ fortissimo; $\mathrm{mf}=$ mezzo-forte; $\mathrm{pp}=$ pianissimo

and A-P laryngeal compression was also conducted. All analysis was performed using Stata 12.1 (StataCorp, College Station, TX). $P<.05$ was considered to be statistically significant, and all reported $P$ values were two-sided.

\section{RESULTS}

Inter-rater agreement controlled by phonatory task and loudness was good (ICC $=0.59,95 \%$ confidence interval $=0.48-0.66, P=.007)$. Tables I through $\mathrm{V}$ show the results (VAS scores) of each one-way ANOVA or $t$ test for univariate analysis of both types of laryngeal compression in relation to loudness, pitch, gender, voice classification, and phonatory task. Male subjects demonstrated a greater degree (higher values) of both A-P $(P=.0001)$ and medial $(P<.0001)$ compression than female subjects. Statistically significant differences between the three loudness levels were also found for medial $(P<.0001)$ and A-P $(P<.0001)$ compression; both types were greatest during fortissimo phonation. Regarding pitch, a significant difference was only demonstrated for medial compression $(P<.0001)$. Both types of compression obtained the highest values during sustained vowel /a/ $(P<.0001)$ and the lowest values during the song $(P<.0001)$. Voice classification also significantly

TABLE III.

Visual Analogue Scale Scores in Relation to Pitch.

\begin{tabular}{llllr}
\hline Score & \multicolumn{1}{c}{ Low } & Middle & High & \multicolumn{1}{c}{$P$} \\
\hline Medial & $13.88 \pm 9.90$ & $15.06 \pm 8.62$ & $17.59 \pm 9.53$ & $<.0001$ \\
$\begin{array}{l}\text { Anterior- } \\
\text { posterior }\end{array}$ & $28.04 \pm 24.28$ & $27.66 \pm 22.80$ & $27.31 \pm 18.56$ & .9339 \\
\hline \hline
\end{tabular}

affected the degree of medial and A-P compression. Tenors demonstrated the greatest value for medial compression. Baritones showed the highest degree of A-P narrowing. Among the women only, sopranos showed the greatest values for medial and A-P compression. Overall medial compression scores demonstrated lower values $(15.09 \pm 9.26)$ than overall A-P compression scores $(26.76 \pm 21.65)$. This difference was statistically significant $(P<.0001)$. Table VI summarizes the results from multivariate regression analysis that considers both laryngeal compression types as dependent variables, and loudness, pitch, gender, voice classification, and phonatory task as predictors. Figure 1 shows the correlation analysis. Medial laryngeal compression significantly correlates with anterior-posterior compression $(r=0.41$; $P<.0001)$

\section{DISCUSSION}

This study was planned and conducted to evaluate the degree of A-P and medial supraglottic laryngeal compression in healthy singers of different voice classifications while singing during different pitches, loudness levels, and phonatory tasks. This study is the first one investigating the effects of these variables on supraglottic laryngeal activity as observed endoscopically. Male sex, loudness, and sustained vowel phonation all were associated with higher VAS scores for both types of hyperfunction. Pitch only affected medial compression scores.

A-P compression has been reported as a regular laryngeal behavior during singing in normal subjects. ${ }^{3,9,11,12}$ Yanagisawa et al. ${ }^{14}$ described A-P compression in singers in the three loudest voice qualities (belting, twang, and opera) and in loud voice segments. The authors reported that during loud voice the tubercle of the epiglottis appeared to move posteriorly, whereas the arytenoids seemed to move anteriorly and/or to tilt caudally as the aryepiglottic sphincter narrowed the vestibule to the larynx. They also demonstrated that there was more acoustic energy associated with a narrowing of the aryepiglottic orifice and less acoustic energy when the epiglottis was elevated and the orifice widened. This is an interesting outcome that is likely to be related to the so-called singer's formant cluster (a concentration of acoustic energy around $3 \mathrm{kHz}$ associated with the "ringing" voice quality). The singer's formant, as described by Sundberg, ${ }^{17}$ may have contributed to the increased acoustic energy associated with anterior-posterior laryngeal compression in the Yanagisawa study. ${ }^{14}$

Another reason that could explain why aryepiglottic narrowing might yield a desirable vocal tract in the singing voice is related to the nonlinear theory of voice

TABLE IV.

Visual Analogue Scale Scores in Relation to Voice Classification.

\begin{tabular}{|c|c|c|c|c|c|}
\hline Score & Soprano & Mezzo & Tenor & Baritone & $P$ \\
\hline Medial & $12.88 \pm 6.93$ & $11.83 \pm 7.44$ & $21.08 \pm 11.65$ & $18.3 \pm 9.36$ & $<.0001$ \\
\hline Anterior-posterior & $25.06 \pm 18.36$ & $24.02 \pm 20.89$ & $19.32 \pm 19.24$ & $37.50 \pm 24.35$ & $<.0001$ \\
\hline
\end{tabular}


TABLE V.

Visual Analogue Scale Scores in Relation to Phonatory Task.

\begin{tabular}{|c|c|c|c|c|c|}
\hline Score & Happy Birthday & $/ a /$ & $/ \mathrm{u} /$ & $/ \mathrm{i} /$ & $P$ \\
\hline Medial & $12.39 \pm 6.94$ & $16.86 \pm 9.18$ & $15.51 \pm 10.34$ & $13.76 \pm 8.63$ & $<.0001$ \\
\hline Anterior-posterior & $19.89 \pm 17.58$ & $37.65 \pm 23.47$ & $22.01 \pm 18.97$ & $21.77 \pm 19.03$ & $<.0001$ \\
\hline
\end{tabular}

TABLE VI.

Multivariate Regression Analysis Considering Both Medial Laryngeal Compression and Anterior-Posterior Laryngeal Compression as Dependent Variables, and Intensity, Pitch, Gender, Voice Classification, and Phonatory Task as Predictors.

\begin{tabular}{|c|c|c|c|c|}
\hline Variables & Coefficients $(95 \% \mathrm{Cl})$ & SE & $t$ & $P$ \\
\hline \multicolumn{5}{|c|}{ Medial laryngeal compression } \\
\hline \multicolumn{5}{|c|}{ Intensity } \\
\hline $\mathrm{pp}$ & 13.64 (12.58 to 14.70$)$ & 0.53 & 25.29 & $<.001$ \\
\hline $\mathrm{mf}$ & $0.80(-0.69$ to 2.29$)$ & 0.76 & 1.06 & .291 \\
\hline $\mathrm{ff}$ & 3.67 (2.16 to 5.18$)$ & 0.76 & 4.78 & $<.001$ \\
\hline \multicolumn{5}{|l|}{ Phonatory task } \\
\hline Happy Birthday song & 12.39 (10.55 to 14.22$)$ & 0.93 & 13.25 & $<.001$ \\
\hline vowel /a/ & 4.47 (2.34 to 6.60$)$ & 1.08 & 4.13 & $<.001$ \\
\hline vowel /u/ & 3.12 (0.95 to 5.29$)$ & 1.10 & 2.83 & .005 \\
\hline vowel /i/ & $1.37(-0.80$ to 3.54$)$ & 1.10 & 1.24 & .216 \\
\hline \multicolumn{5}{|l|}{ Voice classification } \\
\hline Soprano & $12.88(11.80$ to 13.96$)$ & 0.54 & 23.48 & $<.001$ \\
\hline Mezzo & $-1.05(-2.53$ to 0.42$)$ & 0.75 & -1.40 & .163 \\
\hline Tenor & 8.20 (6.38 to 10.01$)$ & 0.92 & 8.87 & $<.001$ \\
\hline Baritone & 5.41 (3.82 to 7.00$)$ & 0.81 & 6.67 & $<.001$ \\
\hline \multicolumn{5}{|l|}{ Pitch } \\
\hline Low & 13.88 (12.74 to 15.02$)$ & 0.57 & 23.99 & $<.001$ \\
\hline Middle & $1.18(-0.42$ to 2.79$)$ & 0.82 & 1.44 & .150 \\
\hline High & 3.71 (2.06 to 5.35$)$ & 0.83 & 4.44 & $<.001$ \\
\hline \multicolumn{5}{|l|}{ Gender } \\
\hline Male & 7.06 (5.88 to 8.25$)$ & 0.60 & 11.74 & $<.001$ \\
\hline Female & $12.32(11.58$ to 13.06$)$ & 0.37 & 32.60 & $<.001$ \\
\hline \multicolumn{5}{|c|}{ Anterior-posterior compression } \\
\hline \multicolumn{5}{|c|}{ Intensity } \\
\hline $\mathrm{pp}$ & 23.30 (20.82 to 25.78$)$ & 1.26 & 18.41 & $<.001$ \\
\hline $\mathrm{mf}$ & $2.54(-0.96$ to 6.05$)$ & 1.78 & 1.42 & .155 \\
\hline $\mathrm{ff}$ & 8.14 (4.59 to 11.68$)$ & 1.80 & 4.51 & $<.001$ \\
\hline \multicolumn{5}{|l|}{ Phonatory task } \\
\hline Happy Birthday song & 19.89 (15.79 to 23.98$)$ & 2.08 & 9.54 & $<.001$ \\
\hline vowel /a/ & 17.75 (13.01 to 22.50$)$ & 2.41 & 7.35 & $<.001$ \\
\hline vowel /u/ & $2.12(-2.70$ to 6.96$)$ & 2.46 & 0.86 & .388 \\
\hline vowel /i/ & $1.88(-2.95$ to 6.72$)$ & 2.46 & 0.76 & .445 \\
\hline \multicolumn{5}{|l|}{ Voice classification } \\
\hline Soprano & 25.06 (22.44 to 27.68$)$ & 1.33 & 18.76 & $<.001$ \\
\hline Mezzo & $-1.04(-4.64$ to 2.55$)$ & 1.83 & -0.57 & .570 \\
\hline Tenor & $-5.73(-10.15$ to -1.32$)$ & 2.24 & -2.55 & .011 \\
\hline Baritone & 12.44 (8.56 to 16.32$)$ & 1.97 & 6.29 & $<.001$ \\
\hline \multicolumn{5}{|l|}{ Gender } \\
\hline Male & 5.84 (2.88 to 8.80$)$ & 1.50 & 3.87 & $<.001$ \\
\hline Female & 24.51 (22.65 to 26.37 ) & 0.94 & 25.90 & $<.001$ \\
\hline
\end{tabular}

$\mathrm{Cl}=$ confidence interval; $\mathrm{ff}$ = fortissimo; $\mathrm{mf}=$ mezzo-forte; $\mathrm{pp}=$ pianissimo; $\mathrm{SE}$ = standard error. 


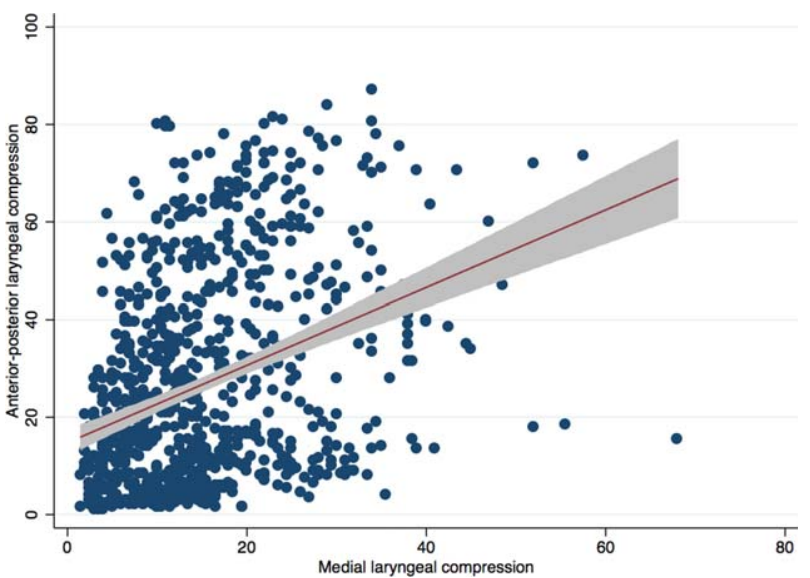

Fig. 1. Correlation analysis between medial and anterior-posterior compression. [Color figure can be viewed in the online issue, which is available at wileyonlinelibrary.com.]

production. According to Titze, ${ }^{18}$ the source-filter interaction and the vocal tract inertance may be increased by narrowing of the epilarynx tube in an A-P direction. Inertance is an acoustic property of the accelerating or decelerating supraglottal air mass in the vocal tract. It may favorably impact vocal fold vibration ${ }^{19}$ and allow for more efficient voice production associated with less effort, more resonance, and more powerful sound. ${ }^{20-22}$

Previous studies have suggested that medial compression also may not in and of itself be pathologic or diagnostic of a voice disorder, but rather may be a dynamic supraglottic activity that is a normal component of articulation at the level of the larynx. ${ }^{3,9,12}$ False vocal fold approximation has been observed in classical singing, as well as other styles. ${ }^{23-25}$ Our findings showed that A-P and medial compression significantly correlated, and therefore occur simultaneously. According to the VAS scores in this study, medial compression showed a significantly lower value than A-P narrowing, suggesting that medial compression does not play as large a role as A-P compression in shaping of the supraglottis in healthy singers. One can extrapolate from this that excessive false vocal fold medial compression might be a sign of pathologic hyperfunction.

Supraglottic hyperfunction was graded as less severe during singing of "Happy Birthday," when compared to sustained vowel phonation. Supraglottic activity seen during sustained vowel production may be referred to as a "static" component of supraglottic activity. ${ }^{8}$ Earlier studies have reported that some sounds promote a more tilted position of the epiglottis and/or a more adducted position of ventricular bands. ${ }^{26}$ Our findings showed that the vowel /a/ produced more medial and A$\mathrm{P}$ supraglottic activity than the other phonatory tasks. Evaluating supraglottic hyperfunction during singing or connected speech is likely more clinically useful than sustained vowel phonation given that standard vocal tract formations are shaped and held for each vowel. However, recognizing which vowels demonstrate more compression could prove useful for the physician or speech pathologist evaluating the patient.
Interestingly, male subjects demonstrated a higher degree of A-P compression than female subjects in the present investigation. A suitable explanation for this outcome could be the degree of laryngeal lowering used to produce a "covered" quality to the sound. In general men, particularly the lower voice types, use a more covered tone than women. Baritones, not surprisingly, had the greatest A-P compression in this study. This hypothesis is in accordance with Sundberg, ${ }^{17}$ who suggested that the lowering of the larynx seems to be a way to obtain greater aryepiglottic narrowing.

Although supraglottic activity may be a normal and even a desirable feature in singing voices, it still may be pathologic in patients with voice disorders. The mean VAS scores in our healthy singer-subjects were $<30$, suggesting that the hyperfunction was felt to be not overly severe. Perhaps patients with voice problems have more severe hyperfunction. Previous studies have reported a lesser degree of A-P compression for normal controls when compared to patients with organic and nonorganic dysphonia. ${ }^{3,9,12}$ Aryepiglottic narrowing is probably a learned pattern that is unconsciously developed throughout the years of singing voice training, or it could also be a natural condition of some talented singers. Conversely, A-P compression of the supraglottis may represent a maladaptive muscular compensatory strategy in subjects with vocal pathology, such as vocal fold paresis. It may be difficult to distinguish maladaptive A-P squeeze from aryepiglottic narrowing. The literature and our study support the notion that some aryepiglottic narrowing is present in the healthy, classically trained singing voice. However, more severe compression may be a sign of pathologic hyperfunction.

Our study is limited in that it only looked at supraglottic activity in healthy singers. There is no comparison group of patients with voice pathology. In addition, we only look at the classically trained operatic singer. The study does not provide information on the healthy speaking voice or other styles of singing. One would expect different supraglottic behavior in other singing styles. Hopefully this study will provide further insight for evaluation of the classically trained singing voice and determining when hyperfunctional behavior is contributing to vocal pathology. Future studies looking at hyperfunction in the healthy speaker, in different singing styles, and in singers or speakers with voice problems will be useful. However, recognizing patterns of supraglottic compression in the healthy trained singer and what variables can affect its severity will hopefully prove useful in furthering our understanding of supraglottic hyperfunction in general. Unfortunately, we were only able to recruit a small number of altos and basses; therefore, we grouped them with mezzo-sopranos and baritones. Ultimately, we do not feel our results would vary much with greater numbers of these voice types, as we still had a wide spectrum of vocal range.

\section{CONCLUSION}

A degree of increased supraglottic activity is seen in the healthy classically trained singing voice. It is 
greatest in males, with louder dynamics, and with sustained vowel phonation. A-P compression is also greater with higher pitch and in baritones. Medial compression is less severe than A-P compression in general, not related to pitch, and greatest in tenors. This information may prove useful in furthering our understanding of supraglottic hyperfunction. Future studies looking at supraglottic activity in other vocal styles, in speaking, and in the pathologic voice are needed.

\section{ACKNOWLEDGMENTS}

The authors thank Suzanne Acton (Michigan Opera Theatre, Detroit and Rackham Symphony Choir, Detroit, Michigan), Dr. Augustus Hill (Brazeal Dennard Choral, Detroit, Michigan), Dr. Emery Stephens (Wayne State University, Detroit, Michigan), Prof. John-Paul White (Oakland University, Rochester, Michigan), and Dr. Ruth Moore (St. John Hospital, Detroit, Michigan).

\section{BIBLIOGRAPHY}

1. Oates J, Winkworth A. Characterising hyperfunctional voice disorders: etiology, assessment, treatment and prevention. Int J Speech Lang Pathol 2008;10:193-194

2. Rubin A, Praneetvatakul V, Gherson S, Moyer C, Sataloff R. Laryngeal hyperfunction during whispering: reality or myth? J Voice 2006;20: 121-127.

3. Stager S, Bielamowicz S, Regnell J, Gupta A, Brakmeier J. Supraglottic activity: evidence of hyperfunction or laryngeal articulation? $J$ Speech Hear Res 2000;43:229-238.

4. Hillman RE, Holmberg EB, Perkell JS, Walsh M, Vaughan C. Objective assessment of vocal hyperfunction: an experimental framework and initial results. J Speech Hear Res 1989;32:373-392.

5. Koufman JA, Blalock PD. Functional voice disorders. Otolaryngol Clin North Am 1991;24:1059-1073.

6. Morrison MD, Rammage LA, Belisle GM, Pullan CB, Nichol H. Muscular tension dysphonia. J Otolaryngol 1983;12:302-306.
7. Roy N, Ford CN, Bless DM. Muscle tension dysphonia and spasmodic dysphonia: the role of manual laryngeal tension reduction in diagnosis and management. Ann Otol Rhinol Laryngol 1996;105:851-856.

8. Dworkin JP, Meleca RJ, Abkarian GG. Muscle tension dysphonia. Curr Opin Otolaryngol Head Neck Surg 2000;8:169-173.

9. Behrman A, Dahl L, Abramson A, Schutte H. Anterior-posterior and medial compression of the supraglottis: signs of nonorganic dysphonia or normal postures? $J$ Voice 2003;17:403-410.

10. Hillman RE, Gress C, Hargrave J, Walsh M, Bunting G. The efficacy of speech-language pathology intervention: voice disorders. Semin Speech Lang 1990;11:297-310.

11. Sama A, Carding PN, Price S, Kelly P, Wilson JA. The clinical features of functional dysphonia. Laryngoscope 2001;111:458-463.

12. Stager S, Bielamowicz SA, Gupta A, Marullo S, Regnell JR, Barkmeier JM. Quantification of static and dynamic supraglottic activity. $J$ Speech Lang Hear Res 2001;44:1245-1256.

13. Lawrence V. Laryngological observations on belting. J Res Singing 1979;2: $26-28$.

14. Yanagisawa E, Estill J, Kmucha S, Leder S. The contribution of aryepiglottic constriction to "ringing" voice quality: a videolaryngoscopic study with acoustic analysis. $J$ Voice 1989;3:342-350.

15. Pershall K, Boone S. Supraglottic contribution to voice quality. $J$ Voice 1987;1:186-190.

16. Hanayama E, Camargo Z, Tsuji D, Pinho S. Metallic voice: physiological and acoustic features. $J$ Voice $2009 ; 23: 62-70$

17. Sundberg J. Articulatory interpretation of the singing formants. $J$ Acoust Soc Am 1974;55:838-844

18. Titze I. Voice training and therapy with a semi-occluded vocal tract: rationale and scientific underpinnings. $J$ Speech Lang Hear Res 2006; 49:448-459.

19. Titze I. Acoustic interpretation of resonant voice. J Voice 2001;15:519-528.

20. Titze I. A theoretical study of F0-F1 interaction with application to resonant speaking and singing voice. $J$ Voice 2003;18:292-298.

21. Titze I. The physics of small-amplitude oscillation of the vocal folds. $J$ Acoust Soc Am 1988;83:1536-1552.

22. Titze I, Story B. Acoustic interactions of the voice source with the lower vocal tract. J Acoust Soc Am 1997;101:2234-2243.

23. Fuks L, Hammarberg B, Sundberg J. A self-sustained vocal ventricular phonation mode: acoustical, aerodynamic and glottographic evidences. KTH TMH-QPSR 1998;3:49-59.

24. Lindestad P, Sodersten M, Merker B, Granqvist S. Voice source characteristics in Mongolian "throat singing" studied with high-speed imaging technique, acoustic spectra, and inverse filtering. J Voice 2001;15:78-85.

25. Hamdan AL, Sibai A, Moukarbel RV, Deeb R. Laryngeal biomechanics in Middle Eastern singing. $J$ Voice 2006;20:579-584.

26. Zeroual C, Esling JH, Crevier-Buchman L. The contribution of supraglottic laryngeal adjustments to voice: phonetic evidence from Arabic. Logoped Phoniatr Vocol 2008;33:3-11. 